\title{
一般口演10
}

\section{The use of quantitative sensory testing in the etiology, diagnosis and management of pain and dysfunction of the masticatory system}

\author{
OIchiro Okayasu, ${ }^{1,2)}$, Kumiko $\mathrm{Oi}^{2)}$ and Antoon De Laat ${ }^{1)}$ \\ ${ }^{1)}$ Department of Oral and Maxillofacial Surgery, School of Dentistry, Oral Pathology and Maxillofacial Surgery, \\ Catholic University of Leuven, Belgium \\ ${ }^{2)}$ Department of Clinical Physiology, Graduate School of Biomedical Sciences, Nagasaki University, Japan
}

\section{I. 目的}

The general theme of my research is to elucidate pathophysiological mechanisms of orofacial pain and to apply these findings in the clinical environment. 1) To objectify and understand the pathophysiology of pain, and contribute to its diagnosis, quantitative sensory testing is of importance ${ }^{1,2)} .2$ ) In addition, combined measurements on both skin and muscle might elucidate to what extent the pain perception results from deep tissues or also from an abnormal superficial perception ${ }^{3)}$. 3) Furthermore, it is known that many factors may modulate sensory and pain perception ${ }^{4,5,6)}$. However, the effect of tooth clenching and/or non-functional tooth contact (which are considered possible risk factors in the development of orofacial pain) on the sensory and pain perception is not investigated up to now.

Consequently, the aim of this study is to examine the effect of light tooth contact, as in diurnal tooth clenching, on the tactile detection threshold (TDT), tactile pain detection threshold (TPDT) and pressure pain threshold (PPT) in the orofacial region.

\section{II. 方 法}

Twenty healthy volunteers (ten men, ten women, age range 23 to 45 years) were recruited. Using a stair-case method, the TDT and the TPDT were measured by means of Semmes-Weinstein monofilaments, on the cheek skin (CS) overlying the masseter muscles (MM) and on the skin overlying the palm side of the thenar muscle (Thenar Skin:
TS). The PPT was measured at the central part of the MM using a pressure algometer. The intensity of pain perceived during TPDT and PPT was scored on a numeric rating scale (NRS).

Each parameter was measured before and after keeping light tooth contact for 5 minutes (session 1) and keeping the jaw relaxed for 5 minutes (session 2) as a control. The two measurements sessions were separated by 1 week and the order randomized.

ANOVA with repeated measures was used to analyze intervention (before and after clenching or no clenching), session (clenching or no clenching) and gender effects for all the parameters (TDT, TPDT, PPT). Post-hoc tests were performed with use of Tukey tests and significance was accepted at $\mathrm{P}<0.05$.

\section{III. 結果·考察}

\section{Tactile detection threshold (TDT)}

Although there were no significant session effects or gender on TDT, there were significant effects of experimental condition on the TDT of left CS (F = 15.92 for male, 23.95 for female, $\mathrm{P}<0.01$ ) and right $\mathrm{CS}$ $(\mathrm{F}=10.20$ for male, 22.25 for female, $\mathrm{P}<0.01)$ and $\mathrm{TS}$ $(\mathrm{F}=9.61$ for male, 12.10 for female, $\mathrm{P}<0.01$ ) in both genders. Post-hoc tests indicated that especially in females, the TDT of CS and TS significantly increased from before to after clenching and/or no clenching (Tukey tests: $\mathrm{P}<0.05$ ).

\section{Tactile pain detection threshold (TPDT)}

Although there were no significant session effects on 
TPDT, there were significant effects of experimental condition on the TPDT of TS in females $(F=10.99, \mathrm{P}<$ 0.01). Males had a significant higher TPDT of left CS $(P<0.05)$ and TS $(P<0.05)$ than females. On the other hand, the NRS for TPDT of CS and TS were significantly higher in females than in males $(P<$ 0.05).

\section{Pressure pain threshold (PPT)}

There were no significant session effects and effects of experimental condition on the PPT. The only significant factor was gender. Males had a significant higher PPT of MM than females $(\mathrm{P}<0.01)$, and there was no significant difference in the NRS for PPT of MM.

Many factors appear to modify the sensory and pain perception, e.g., attentional influences ${ }^{4)}$, motor activity $^{5)}$ and movement ${ }^{6}$. Feine et al ${ }^{5)}$ used thermal stimuli applied to the limb and tested the effect of movement on the perception of pain. They found that motor activity decreased the ability to detect weak low-threshold cutaneous inputs, but had no effect on the perception of warmth and heat pain. Chapman et al ${ }^{6}$ used electrical stimulation and tested the sensory perception during movement of the stimulated arm. They also found that the perceived intensity of a suprathreshold stimulus was not altered by movement while the detection thresholds were increased during movements. These results suggest that elevation of sensory and pain thresholds could be explained by masking, which has a greater effect on sensory threshold than on pain threshold.

In our study, the modulation of the TDT might thus result from habituation rather than from tooth contact, but it was striking that the changes seem to be more present in women than men. In the next project, we plan to examine whether these effects are similar in patients suffering from myofascial pain in the jaw muscles. If this is the case, a different physiological reaction in patients could be conceptualized, which might later on be used to identify better subjects at risk to develop pain and dysfunction ${ }^{7}$.

\section{IV. 文 献}

1) Jacobs R, Wu CH, Van Loven $\mathrm{K}$, Desnyder M, Kolenaar B, Van Steenberghe D.: Methodology of oral sensory tests, J Oral Rehabil, 29: 720-730, 2002.

2) De Laat A, Stappaerts K, Papy S.: Counseling and physical therapy as treatment for myofacial pain of the masticatory system, J Orofacial Pain, 17: 42-49, 2003.

3) Komiyama O, De Laat A.: Tactile and pain thresholds in the intra- and extra-oral regions of symptom-free subjects, Pain, 115: 308-315, 2005.

4) Bushnell MC, Duncan GH, Dubner R, Jones RL, Maxiner W.: Attentional influences on noxious and innocuous cutaneous heat detection in humans and monkeys, J Neurosci, 5: 1103-1110, 1985.

5) Feine JS, Chapman CE, Lund JP, Duncan GH, Bushnell MC.: The perception of painful and nonpainful stimuli during voluntary motor activity in man, Somatosens Mot Res, 7: 113-124, 1990.

6) Chapman CE, Bushnell MC, Miron D, Duncan GH, Lund JP.: Sensory perception during movement in man, Exp Brain Res, 68: 516-524, 1987.

7) Michelotti A, Farella M, Stellato A, Martina R, De Laat A.: Tactile and pain thresholds in patients with myofascial pain of the jaw muscles; A case-control study, J Orofacial Pain, 22: 139-145, 2008. 